\title{
CCD PHOTOMETRY - PRESENT AND FUTURE
}

\author{
Alistair R. Walker \\ Cerro Tololo Inter-American Observatory
}

\begin{abstract}
I will briefly treat some of the developments relating to CCD photometry which have taken place over the last two to three years, and speculate on those which can be anticipated to take place in the near future. CCDs have been in widespread use as astronomical detectors for slightly more than a decade. For the majority of visual-light projects they have displaced the photographic plate and the photomultiplier as the detector-of-choice, except in applications requiring large area coverage or high time resolution, but even here the development of arrays and the use of more sophisticated electronics has permitted encroachment into these domains. The use of large, low-noise detectors for CCD photometry places stringent demands on telescope optics, instruments, controllers, calibration procedures, data reduction methods and data storage, and forces a holistic approach to managing the data flow from detector to final storage medium.
\end{abstract}

For an earlier review on this field see Walker (1993); other papers presented at IAU Colloquium No. 136 and IAU Symposium 167 (these proceedings) should be consulted for different topics, alternative viewpoints, and more in-depth discussions of specific subjects.

\section{THE PRESENT}

Changes in the last two to three years include:

\subsection{More General Availability of High-Performance CCDs}

For an over-all summary see P. Jorden (1995). Concentrating on devices used for direct imaging, SITe (previously Tektronix) are delivering, albeit at a rather slow rate, thinned CCDs of high cosmetic quality with format $2048 \times 2048$ and 24 micron pixels. A significant fraction of these CCDs have four working amplifiers, and perhaps their only problem is that the CCD surface has a bow such that the center is some 250 microns higher than the corners. This is significant when these CCDs are used in fast beams. For a dedicated dewar the window could be replaced by a suitable "field-deflattener" lens, and this approach has been successful when using these CCDs in very fast spectrograph cameras. Together with their smaller brethren (1024 $\mathrm{x} 1024,512 \times 512$ ), the SITe CCDs are near-ideal for direct imaging. They do have quantum efficiency response that falls off below $4000 \AA$, but are much improved in this respect when compared to Tektronix CCDs produced four to five years ago. Typical QE figures are $25 \%$ at $3000 \AA$ and $60 \%$ at $4000 \AA$. CCDs with the same number of pixels have been obtained via foundry runs at Loral (previously Ford Aerospace), but the number of thinned versions that have been produced is extremely small. The same is true of other producers and it is clear that without Tektronix CCDs photometrists would be very much disadvantaged. 
Another important development is that manufacturers are now usually fabricating CCDs with MPP (multi-pin phase) implants. When a CCD is clocked in MPP mode the dark rate at a given temperature is lower than in non-MPP mode, at the expense of lowering full-well capacity. Although to achieve dark rate floors of a few $\mathrm{e}^{-} /$hour it is still necessary to cool to typically $-100^{\circ} \mathrm{C}$, MPP CCDs achieve dark rates at temperatures $-40^{\circ} \mathrm{C}$ to $-60^{\circ} \mathrm{C}$ that are still low enough that dark noise is not the major noise source for exposures of up to a few minutes. At these temperatures Peltier coolers rather that liquid cryogens can be used, and several systems based on this technology are on the market. Using relatively inexpensive frontilluminated CCDs, they seem particularly suitable for those not requiring an expensive state-ofthe art system and indeed there are many scientifically valuable programs (e.g. variable stars, supernovae searches) for which such a system is entirely adequate. It is perhaps unfortunate that the emphasis in the amateur realm, particularly in the USA, seems mostly aimed at producing pretty pictures.

\subsection{Higher Performance CCD Controllers}

A new generation of CCD controllers is appearing (R. Leach 1995 and poster papers, these proceedings). These offer many advantages. They are more reliable due to lower component count and the use of modern packaging techniques such as multi-layer boards and surface mounting of components. Performance has improved due to better op-amps, faster, more accurate, and self-calibrating Analog-to-Digital convertors (ADCs) and the utilization of fiber optics to aid isolation. Power consumption need only be a few watts. Typically, each unit contains four separate video paths to accommodate CCDs with multiple amplifiers, and include the large number of clock and bias levels needed to be able to accommodate the possible readout schemes. In general, these controllers incorporate powerful processors (DSP's, transputers) which can provide significant computing power in the controller itself. This can be used for pre-processing the data (essential for IR arrays) or, for instance, to control a flexible real-time display (Walker 1995). Making these controllers compact and reliable as well as delivering state-of-the-art noise performance is not a trivial task and, so as not to degrade the effectiveness of the hardware, the software tends to be both complex and extensive. This can have distinct advantages, for instance sophisticated software tools can aid development (eg CCD waveform generation tools), and can easily control DACs (Digital to Analog convertors) to provide programmable voltages which, apart from initially setting the bias and clock levels, can monitor these levels to make sure they are always correct. It is relatively simple to incorporate a DAC to provide controllable video gain, and so on. The user can take advantage of this flexibility in that it is possible to tailor the CCD data acquisition system to be optimal for a specific program. A corollary of this flexibility is that the detailed characteristics of such a CCD system tend to be less well-known. Photometry is difficult enough already without adding a poorly-characterized detector system to the list of the observer's woes, and a wiser approach would seem to be to identify a small number of options that are adequate for the great majority of programs, and characterize these modes of operation in detail.

\subsection{Better Data Acquisition Software, More Powerful Computers}

Over the past decade the improvement in computer processing power has more than kept pace with the increase in numbers of pixels of a typical CCD (Blecha 1995). Computer memory technology, together with disk and tape storage, has also advanced in similar fashion. The efficient processing and storage of data from detectors such as the SITe 2048's is, if not 
something to be dismissed as a minor problem, certainly soluble in terms of hardware that costs very much less than the detector itself. At the same time, the data acquisition process has become more efficient for the user, by provision of easily-learned user interfaces and the integration of observation and reduction processes. High band-width networks allow multiple observers to share, possibly remotely, observation and reduction tasks. It is feasible to ascertain the quality of the incoming data in near real-time by examining image focus, measuring signal/noise, doing aperture photometry of standard stars, etc. This ability has a real effect on the quality of data, for too long have many CCD photometrists tolerated data acquisition processes that have given them far less feed-back on their data than typically enjoyed by those doing photo-electric photometry.

\subsection{HST}

The resolution of better than 0.1 arcsec, as a result of the highly successful 1993 refurbishment mission, is resulting in deeper photometry than ever achieved from the ground, and is allowing work in crowded fields impossible to study effectively even with the best ground-based telescopes. However some care must be taken. HST images are poorly- or undersampled, even on the high-resolution PC CCD where images with $\mathrm{fwhm}=1.8$ pixels are routinely achieved. the star-cosmic ray differentiation must be done with care (Windhorst et al. 1994). Since the Loral CCDs used on HST WFPC-2 are front-illuminated, there will be considerable intra-pixel response variations (Jorden et al. 1994). Thus for best results exposures should be split and the telescope jogged slightly between each individual exposure to randomize the position of any given star with respect to its distance form a pixel center. Given these precautions, and by applying standard reduction techniques, excellent photometry can be obtained by conventional aperture or psf photometry. Although the STScI provides absolute calibration figures for the CCD-filter combinations, a safer and, if properly done, an inherently more accurate technique is to perform the absolute calibration via ground-based observations.

This need not require a large telescope. Another aspect to be considered is that the WFPC broad-band filters are not particularly good reproductions of UBVRI. Ground-based comparisons for many of the filters appear in Harris et al. (1993), see also Harris et al. (1991) and Walker (1994), and from these data it is in principle possible to transform HST measurements to the standard system. An alternative procedure is to calibrate via ground-based observations using reproductions of the WFPC filters, and then derive the requisite astrophysical parameters via isochrones or by theoretical fluxes obtained by convolving model atmospheres with the filter passbands.

\subsection{Standard Stars, and Photometric Calibrations}

As far as broad-band UBVRI observers are concerned, the work by Landolt (1992) is of prime importance. He provides photometry for many stars in the (equatorial) Selected Areas and also in a number of convenient, CCD-sized fields. The latter generally consist of fields chosen to contain a blue star and a number of redder stars in order to provide stars of similar magnitude but with a wide range in color. The number of stars of magnitudes $10-15$ with accurate photometry is extensive, and this work is of the utmost value, since it allows many more standard stars to be observed for a given integration plus read-time. 
For standard star work the read-time overhead is often dominant when compared to integration time, particularly for large CCDs on moderate to large telescopes. It is has certainly been the case in the past that CCD imaging projects done on large telescopes have been inadequately calibrated due to a perceived large overhead of time required to do the calibration work. This is no longer the case. Many of the stars in the Landolt (1992) lists have rather few observations, and the extensive CCD observations that have been made of these fields can in principle be collated and improved magnitudes derived. Work of this nature is in progress (P. Stetson, private communication). Menzies (1992) has emphasized that the differing implementations of the Johnson UBV system is worrying, and differences can be large, especially for stars of extreme colors, and for (U-B) in general. We should also mention the useful bibliographic list of UBVRI sequences (Ritzmann 1992). An important minority of CCD photometry uses filters other than UBVRI. An important subset of observations are made using narrow-band filters to study emission-line sources such as supernovae remnants and $\mathrm{H}$ II regions. Hamuy et al. $(1992,1994)$ provide a new, accurate set of spectrophotometry for the southern secondary and tertiary standards.

Specialized systems such as Gunn, Washington, Strömgren, DDO, Geneva and Vilnius are used by a minority of CCD observers, in general to provide sharper discrimination of astrophysical quantities (e.g., temperature, metallicity, gravity) than is possible with the UBVRI system. The conference references mentioned above provide several papers devoted to work using these systems, an example of their usefulness can be gauged from the paper by Geisler et al. (1991) which provides the metal abundance calibration from Washington photometry, while examples of precision work on standard stars are (for DDO) provided by Cousins (1993) and (for Strömgren) by Kilkenny and Laing (1992).

\subsection{Larger Telescopes, Improved Image Quality}

The great majority of CCD imaging projects would benefit from improved image quality, with the few exceptions including surface photometry of large clusters or galaxies, programs where only bright, isolated stars are measured, and cases where the pixel size is such that the images are already under-sampled in moderate seeing. The problem is two-fold. Firstly, seeing monitor measurements show that many mountain-top observatories enjoy median seeing of typically $\sim 0.7$ arcsec and this is then often ruined by some combination of the telescope optics and the telescope environment. Examples such as the ESO NTT telescope at La Silla and the WIYN telescope at Kitt Peak show that telescopes designed with stringent imaging errorbudgets can indeed achieve close to the site seeing, and this approach is being carried out even more carefully for the new generation of very large telescopes. The example of the CFHT shows that improvements to a previous generation of telescopes are indeed possible and can produce similar performance. Various papers in Stepp (1994) are a useful introduction to the literature, as well as describing in detail both the performance goals of the new generation of very large 8 to $10-\mathrm{m}$ class telescopes and programs for improving already existing telescopes. Smaller (1 to 2-m) class telescopes are often used for CCD imaging programs and can also benefit from such upgrades. For these, replacement of optics, often made in an age where seeing was measured to one significant figure only, can be advantageous and need not be financially crippling. 


\section{THE NEAR FUTURE}

\subsection{Large Multi-Amplifier CCDs}

There will be more general availability of large multi-amplifier CCDs. Manufacturers such as EEV, Reticon, SITe, and Thomson are actively pursuing the scientific market, while custom devices can be from procured from Loral, Orbit and some of the manufacturers just mentioned. The successful thinning of CCDs is still a black art which few have mastered, and for which the user pays a substantial premium; however gains of two to three in blue QE and 1.5 in the red are very substantial. Anti-reflection coating technology improvements will allow the option of optimizing for higher $\mathrm{QE}$ at the ends of this optical range, with only minor trade-offs elsewhere. Amplifier design improvements should allow lower read times without compromising read noise. A more general worry for the future is that most of the CCD manufacturers rely on outdated four to five inch diameter wafer fabrication lines for which it is becoming increasingly difficult to buy high-quality silicon. The size of the scientific CCD market alone precludes the substantial investment needed to set up a modern fabrication line using larger wafers.

\subsection{Mosaics of CCDs}

Mosaics of CCDs will provide fields of view comparable to those that have been obtainable using photographic plates. This will lead to a renaissance of wide-field imaging projects, of two general types. The first is the study of large structures, such as distant clusters of galaxies, and near-by galaxies. The second type is deep surveys for rare objects, such as high red-shift quasars, field-star studies in our galaxy and in the Magellanic Clouds. CCD controllers able to operate mosaics will be constructed from the new generation of extensible controllers which are just now appearing. The advance of computer technology will mean that the problems of processing and storing gigabytes of data per night will be surmountable. Projects such as MACHO, which uses a mosaic of four 2048 x 2048 CCDs and pipelines the data-taking process through to the photometry of the stars observed, are already showing the feasibility of wide-field imaging projects. Projects using much larger arrays (e.g., the Sloan Digital Sky Survey) are in the planning or construction phase. These large CCDs place critical demands on the quality of telescope optics, and telescopes optimized for wide-field imaging are a prerequisite. The use of Schmidt telescopes with relatively coarse pixels scales will allow surveys to cover fields of several square degrees at a time to greater depths than has been possible photographically. For extended objects the detection limit for a given pixel size is dependent only on $\mathrm{f} /$ ratio, and small Schmidt telescopes can be surprisingly competitive.

\subsection{Improved Quality of Photometry}

Improved quality of photometry will result from attention to bandpass-matching and the provision of more (and fainter) standards. Further work on modeling the behavior of photometric systems by convolution of theoretical fluxes with the filter-detector bandpasses (e.g., Bell et al. 1994) will help in the astrophysical interpretation of photometry. However the over-subscription of the larger telescopes at national facilities which has resulted in small time allocations per investigator has often, judging from the published literature, caused data to be published where the attention to standardization has been less than adequate. 
This is a great pity since the CCD has shown itself to be capable of highly accurate results. It is suggested that investigators always propose to carry out a supplementary calibration program on a smaller telescope to support observations made on a larger one. It is, unfortunately, necessary to reiterate that one cannot expect to do good CCD photometry in non-photometric conditions, and the widely practiced technique of exposing through thin cloud has dubious worth if accurate photometry is desired. Thin cloud is rarely uniform over the field of a large $\mathrm{CCD}$, even when integrated for many minutes.

\subsection{Reduction Software}

Reduction software will need to keep pace with mosaics, and in particular will need to run in memory to minimize slow input/output steps. The initial bias subtraction, flatfielding and geometric transformations will require powerful data acquisition systems with lots of memory, disk storage, and efficient tape or optical disk storage. Similar machines will be required to make operation of programs such as DoPhot and DAOPHOT run efficiently. Such systems (e.g., SparcStation 20, $512 \mathrm{MB}$ memory, $30 \mathrm{~GB}$ disk system, fast display, double exabyte) cost under $\$ 100,000$, and can typically process (trim, bias subtract, flatfield, register) a typical night's worth of data ( $\sim 25 \mathrm{~GB}, 100$ images) from an $8 \mathrm{k} \mathrm{x} 8 \mathrm{k}$ mosaic in just a few hours (S. Grandi, 1994). Certainly even more capable machines will be available for a similar price in the near future, however it is very clear that the sheer quantity of data produced will require careful attention to data management aspects. The software effort needed to minimize the time between acquisition and processed data will be considerable. Schemes such as the KPNO "Save-the-bits" (Pilachowski et al. 1993) automatically back-up raw data frames immediately following read-out, which apart from removing the onus of storing raw data from the observer serves as a data archive.

\subsection{Adaptive Optics}

Adaptive optics is a new and exciting way to improve the resolution of ground-based observations, over a small field. Development of the technology is rapid (Ealey and Merkle 1994), following the military declassification of the basic techniques. A challenge for the future is to develop the means for making photometric measurements on CCD frames for which adaptive techniques have been used to sharpen the star images. Variable-PSF methods have been very successful at modeling star images on CCD frames where aberrations cause star profile variations as a function of field position. The need to be able to do photometry on aberrated HST CCD frames spawned many new and modified techniques, with varying degrees of success. The challenge for the future will be to refine these methods in order that reliable photometry can be achieved on frames where the star profiles are two-component (sharp peak, plus low intensity wings) and vary with field position.

\section{REFERENCES}

Bell, R. A., Paltaglou, G. and Tripicco, M. J. 1994 MNRAS 268, 771

Blecha, A. 1995 in IAU Symposium No. 167, New Developments in Array Technology and Applications, A. G. D. Philip, K. A. Janes and A. R. Upgren, eds, Kluwer Academic Press, Dordrecht, p. 57

Cousins, A. W. J. 1993 SAAO Circ. 15, 30

Ealey, M. and Merkle, F., editors 1994 Adaptive Optics in Astronomy, Proc. SPIE 2201 
Geisler, D., Claria, J. J. and Minniti, D. 1991 AJ 102, 1936

Grandi, S. 1994, NOAO internal report

Hamuy, M., Walker, A. R., Suntzeff, N. B., Gigoux, P., Heathcote, S. R. and Phillips, M. M. 1992 PASP 104, 533

Hamuy, M., Suntzeff, N. B, Heathcote, S. R., Walker, A. R., Gigoux, P. and Phillips, M. M. 1994 PASP 106, 566

Harris, H. C., Baum, W. A., Hunter, D. A. and Kreidl, T. J. 1991 AJ 101, 677

Harris, H. C., Hunter, D. A., Baum, W. A. and Jones, J. H. 1993 AJ 105, 1196

Jorden, P. R. 1995 in IAU Symposium No. 167, New Developments in Array Technology and Applications, A. G. D. Philip, K. A. Janes and A. R. Upgren, eds, Kluwer Academic Press, Dordrecht, p. 27

Jorden, P. R., Deltorn, J. -M. and Oates, A. P. 1994 Instrumentation in Astronomy VIII, D. L. Crawford and E. R. Craine, eds., Proc. SPIE 2198, p. 836

Kilkenny, D. and Laing, J. D. 1992 MNRAS 255, 308

Landolt, A. U. 1992 AJ 104, 340

Leach, R. 1995 in IAU Symposium No. 167, New Developments in Array Technology and Applications, A. G. D. Philip, K. A. Janes and A. R. Upgren, eds, Kluwer Academic Press, Dordrecht, p. 49

Menzies, J. W. 1992 in IAU Colloquium No. 136, Stellar Photometry - Current Techniques and Future Developments, C. J. Butler and I. Elliot, eds., Cambridge Univ. Press, Cambridge, p. 40

Pilachowski, C., Seaman, R. and Bohannan, B. 1993 NOAO Newsletter 35, 32

Ritzmann, B. M. 1992 Bull. Inf. CDS, 40, 41

Stepp, L. M., editor 1994 Advanced Technology Optical Telescopes V, Proc. SPIE 2199

Walker, A. R. 1993 in IAU Colloquium No. 136, Stellar Photometry - Current Techniques and Future Developments, C. J. Butler and I. Elliot, eds., Cambridge Univ. Press, Cambridge, p. 278

Walker, A. R. 1994 PASP 106, 828

Walker, A. R. 1995 in IAU Symposium No. 167, New Developments in Array Technology and Applications, A. G. D. Philip, K. A. Janes and A. R. Upgren, eds, Kluwer Academic Pub., Dordrecht, p. 339

Windhorst, R. A., Franklin, B. E. and Neuschaefer, L. W. 1994 PASP 106, 798

\section{DISCUSSION}

HOG: I often miss the information on QE curves whether the CCD is thin or thick. Why not write it?

WALKER: Thick CCDs are the ones with peak QE less than 50\%.

PEL: Do you know anything about the prospects for interference filter layers deposited directly onto the chip?

WALKER: Most thinned CCDs are coated with single layer anti-reflection coatings. Two layer coatings (by Mike Lesser, U. of Arizona) show excellent $\mathrm{QE}$ throughout the whole optical range, for thinned UV-floated CCDs. 
WATSON: Do you use a field-flattener in the Schmidt telescope with 60 arc-min field coverage?

WALKER: No, but certainly for any CCD or mosaic larger than this curvature of field is a problem that will need to be solved either with a field-flattener or by constructing the mosaic such that it conforms to the focal surface.

COOK: What is the opening/closing time for the shutter of the proposed $8 \mathrm{k} \mathrm{x} 8 \mathrm{k}$ mosaic?

WALKER: A curtain shutter will be used. This type of shutter has, by design, equal exposure lines everywhere on the mosaic even for very short (eg. second) exposure times.

PENNY: The faintest stars in the HST CMD you showed seemed to have quite small errors. I presume that there were fainter stars with the expected increase in errors as you go fainter. Was there a reason why these stars were not shown?

WALKER: The CMD was a preliminary one and the processing is not yet complete. The faintest stars were not shown.

O'DONOGHUE: Does intra-Pixel QE variability discovered by the RGO group apply to thinned, back-illuminated CCDs or did Paul Jorden say the problem applied to thinned chips but (perhaps puzzlingly) front-illuminated. I know I should be asking this to Paul Jorden.

WALKER: Inter-Pixel QE variations are more significant for front-illuminated (thick) CCDs than they are for back-illuminated (thinned) CCDS.

JORDEN: To clarify - we measured a thick, frontside, coated EEV device and a thin, backside TEK device. Contact me, or see references for more details (Jorden et al. 1974, Proc SPIE 2198, 836). 